\title{
Activity of the Novel Succinate Dehydrogenase Inhibitor Fungicide Pydiflumetofen Against SDHI-Sensitive and SDHI-Resistant Isolates of Botrytis cinerea and Efficacy Against Gray Mold
}

\author{
Leiming He, ${ }^{1}$ Kaidi Cui, ${ }^{1}$ Yufei Song, ${ }^{1}$ Tongtong $\mathrm{Li},{ }^{1}$ Ning Liu, ${ }^{2}$ Wei Mu, ${ }^{1}$ and Feng Liu ${ }^{1, \dagger}$ \\ ${ }^{1}$ Shandong Provincial Key Laboratory for Biology of Vegetable Diseases and Insect Pests, College of Plant Protection, Shandong \\ Agricultural University, Tai'an, Shandong 271018, People's Republic of China \\ 2 Department of Mycology, College of Plant Protection, Shandong Agricultural University, Tai'an, Shandong 271018, People's \\ Republic of China
}

\begin{abstract}
Succinate dehydrogenase inhibitor (SDHI) fungicides are currently the most frequently used fungicides for controlling gray mold. However, isolates of Botrytis cinerea resistant to SDHI fungicides have emerged in the field. Pydiflumetofen is a new SDHI fungicide that can control a variety of fungal diseases, but its efficacy against gray mold and whether the activity of pydiflumetofen is affected by the current SDHI-resistant isolates is currently unknown. The sensitivity of 291 single-spore $B$. cinerea isolates collected from 2017 to 2019 to pydiflumetofen was determined by spore germination inhibition assays. The mean $\mathrm{EC}_{50}$ value (fungicide concentration resulting in a $50 \%$ inhibition compared with that of the control) of pydiflumetofen was $0.06 \pm 0.01,0.07 \pm 0.02$, and $0.05 \pm$ $0.02 \mathrm{mg} / \mathrm{liter}$ in 2017,2018 , and 2019, respectively. There was no significant difference in the sensitivity of $B$. cinerea to pydiflumetofen among

superior to that of boscalid at $400 \mathrm{mg} /$ liter $(42.7 \%)$. The isolates carrying P225F, N230I, H272Y, and H272R mutations in the $S d h \mathrm{~B}$ subunit were associated with the less sensitivity of $B$. cinerea to SDHI fungicides. After establishing the baseline sensitivity of $B$. cinerea to pydiflumetofen $\left(\mathrm{EC}_{50}\right.$ of $0.03 \pm 0.003 \mathrm{mg} / \mathrm{liter}$ ), we found that the $\mathrm{P} 225 \mathrm{~F}$ and $\mathrm{H} 272 \mathrm{Y}$ mutant isolates showed low to moderate levels of resistance to pydiflumetofen, and the H272R and N230I mutant isolates showed low levels of resistance. The reduced sensitivity to pydiflumetofen resulted from the positive correlation of pydiflumetofen with the other four SDHI fungicides (i.e., boscalid, fluopyram, isopyrazam, and benzovindiflupyr). These results suggest that pydiflumetofen provides effective control for the management of gray mold but must be used with caution.
\end{abstract} the 3 years. Furthermore, pydiflumetofen at $300 \mathrm{mg} /$ liter effectively controlled gray mold on cucumber leaves $(80.9 \%)$, and its efficacy was
Keywords: baseline sensitivity, boscalid, Botrytis cinerea, pydiflumetofen
Gray mold, caused by Botrytis cinerea Pers.:Fr. (teleomorph Botryotinia fuckeliania), is a common disease of vegetables and fruits (Zheng et al. 2015), and its management mainly relies on chemical control (Fan et al. 2017). However, B. cinerea is known as a classical "high-risk" pathogen because of its extensive host range and high genetic variability (He et al. 2018a). Indeed, B. cinerea has developed resistance to many categories of fungicide, such as benzimidazoles, anilinopyrimidines, and dicarboximides. This makes it very difficult to effectively manage gray mold, and it is urgent to find a highly active rotational fungicide.

The action of succinate dehydrogenase inhibitors (SDHIs) on mitochondrial succinate dehydrogenase (SDH) complex II blocks cellular energy transfer, thereby inhibiting the development of pathogens in the field (Fraaije et al. 2012). As a result of its broad spectrum against fungi, this class of compounds has developed rapidly since the advent of boscalid (Lalève et al. 2014). To date, 18 SDHI fungicides, such as fluopyram, isopyrazam, and benzovindiflupyr, have been introduced to the market to control numerous phytopathogenic fungi (Hu et al. 2016). However, only boscalid and penthiopyrad have been registered in China for controlling gray mold (China

${ }^{\dagger}$ Corresponding author: F. Liu; fliu@sdau.edu.cn

Funding: This work was supported by a grant from the National Key Research Development Program of China (2016YFD0200500).

*The $\boldsymbol{e}$-Xtra logo stands for "electronic extra" and indicates that supplementary materials are published online.

The author(s) declare no conflict of interest.

Accepted for publication 21 February 2020.

(C) 2020 The American Phytopathological Society
Pesticide Information Software). Pydiflumetofen is a new SDHI fungicide developed by Syngenta (Buxton et al. 2016); to date, there are numerous reports of the effective controls of pydiflumetofen against Fusarium asiaticum and Sclerotinia sclerotiorum (Duan et al. 2019; Hou et al. 2017). However, no detailed information was reported on the activity of pydiflumetofen on $B$. cinerea. Furthermore, the frequent use of SDHI fungicides against $B$. cinerea has led to serious resistance worldwide (Bardas et al. 2010; Kim and Xiao 2010; Wang et al. 2015). Previous studies have found that the resistance of B. cinerea to SDHIs is correlated with mutations at amino acids 225 (P225L/F/T), 230 (N230I), and 272 (H272Y/R/L) of the $S d h B$ subunit (Yin et al. 2011), and different mutant isolates exhibit different degrees of resistance to a variety of SDHI fungicides such as fluopyram, isopyrazam, and fluxapyroxad (Veloukas et al. 2013). Whether the activity of pydiflumetofen on $B$. cinerea is affected by current SDHI-resistant isolates is unknown.

Therefore, the objectives of this study were as follows: (i) to assess the sensitivity of $291 \mathrm{~B}$. cinerea isolates to pydiflumetofen collected in Shandong Province from 2017 to 2019, (ii) to evaluate the efficacy of pydiflumetofen on detached tomato fruits inoculated with SDHIsensitive and SDH-resistant isolates, (iii) to determine the correlation between pydiflumetofen and the other four SDHI fungicides (i.e., boscalid, fluopyram, isopyrazam, and benzovindiflupyr), and (iv) to examine the efficacy of pydiflumetofen on cucumber leaves against gray mold in a greenhouse.

\section{Materials and Methods}

Fungicides. Technical-grade pydiflumetofen (95\% active ingredient [a.i.]; Syngenta China Investment Co., Ltd.), fluopyram (96\% a.i.; Bayer Crop Science), benzovindiflupyr (97\% a.i.; Syngenta China Investment Co., Ltd.), boscalid (96\% a.i.; Zhejiang Heben Technology Co., Ltd.), and isopyrazam ( $92 \%$ a.i.; Syngenta Switzerland Crop Protection Co., Ltd.) were dissolved in methanol to prepare stock solutions, which were stored at $4{ }^{\circ} \mathrm{C}$ in the dark for use 
in in vitro sensitivity assays. Formulated pydiflumetofen $(200 \mathrm{~g} / \mathrm{liter}$ SC; Syngenta China Investment Co., Ltd.) and boscalid (Cantus 50\% WG; BASF China Co., Ltd.) were used in the detached tomato assay and greenhouse experiment.

Fungal isolates and sequencing of the $S d h B$ gene. Diseased vegetables with characteristics of $B$. cinerea infection were collected from 10 regions in the Shandong Province of China. A total of $291 \mathrm{~B}$. cinerea isolates (91 in 2017, 93 in 2018, and 107 in 2019) were isolated and identified by strict procedures (morphology, genealogical, biochemical, molecular, etc.) according to a previously described method (Fernández-Ortuño et al. 2012). These isolates were stored at $4^{\circ} \mathrm{C}$. According to the method of Leroux et al. (2010), the DNA of the 291 isolates was extracted using the Fungal DNA Mini Kit (Omega Bio-Tek, Doraville, GA) and sequenced on the $S d h B$ gene to identify the genetic background. Forward and reverse PCR primers $\left(5^{\prime}\right.$ CCACTCCTCCATAATGGCTGCTCTCCGC- $3^{\prime}$ and (5'-CTCAT CAAGCCCCCTCATTGATATC- $3^{\prime}$, respectively) were designed to amplify gene encoding for the $S d h \mathrm{~B}$ subunit (GenBank accession KR866382.1). PCR amplifications were performed in a 50- $\mu$ l reaction mixture containing $5 \mu \mathrm{l}$ of $10 \times$ EasyTaq DNA polymerase buffer, $4 \mu \mathrm{l}$ of dNTP, $2 \mu l$ of DNA template, $1 \mu l$ of each primer, $0.5 \mu l$ of EasyTaq DNA polymerase, and $36.5 \mu \mathrm{l}$ of $\mathrm{ddH}_{2} \mathrm{O}$. Amplification conditions consisted of $94^{\circ} \mathrm{C}$ for $90 \mathrm{~s}$; followed by 30 cycles at $94^{\circ} \mathrm{C}$ for $20 \mathrm{~s}, 56^{\circ} \mathrm{C}$ for $20 \mathrm{~s}$, and $72^{\circ} \mathrm{C}$ for $60 \mathrm{~s}$; and a final extension at $72^{\circ} \mathrm{C}$ for $5 \mathrm{~min}$. The PCR products were visualized by electrophoresis on a $1.0 \%$ agarose gel run in $1 \times$ TAE buffer and then purified using the EasyPure Quick Gel Extraction Kit (TransGen Biotech, China). The purified products were cloned into the pGEM-T vector (Huayueyang Biotechnology Co., Ltd., Beijing, China) and sequenced by Shanghai Sangon Biological Engineering Technology and Service Co., Ltd. (China). DNAMAN (version 6.0; Lynnon Biosoft, Quebec, Canada) was used to compare the sequences of the amplified fragments.

Sensitivity of $B$. cinerea to pydiflumetofen in vitro. According to the previously described method (Song et al. 2016), the sensitivity of $B$. cinerea to pydiflumetofen was determined by spore germination inhibition assays. The spore germination inhibition assay was performed on yeast peptone acetate (YBA) medium (10 g of yeast extract, $10 \mathrm{~g}$ of Bacto peptone, $20 \mathrm{~g}$ of sodium acetate, and $15 \mathrm{~g}$ of agar per liter of distilled water) with final concentrations of pydiflumetofen at $0,0.001,0.005,0.01,0.05,0.1,0.5$, and $1 \mathrm{mg} /$ liter. Plates amended with equal volumes of methanol were used as controls. The $\mathrm{EC}_{50}$ value (the fungicide concentration resulting in a $50 \%$ inhibition compared with that of the control) was calculated for each isolate. Three replicate plates were used for each treatment, and all experiments were performed three times. Resistance factors (RFs) were calculated by dividing the $\mathrm{EC}_{50}$ value of resistant isolates by the mean $\mathrm{EC}_{50}$ value of sensitive isolates. According to Veloukas et al. (2011), the following criteria were used for isolate classification: $10>\mathrm{RFs}>2$, isolate with low resistance; $100>\mathrm{RFs}>10$, isolate with moderate resistance; and RFs $>100$, isolate with high resistance.

Efficacy of pydiflumetofen on detached tomatoes inoculated with SDHI-sensitive and SDHI-resistant isolates. According to the method of Veloukas et al. (2013), 15 B. cinerea isolates were selected to determine the efficacy of pydiflumetofen on detached tomato fruits. Three isolates of the sensitive, P225F, N230I, H272Y, and H272R mutants were used (Supplementary Table S1). The most commonly used SDHI fungicide, boscalid, was used as a control fungicide. Tomato fruits with similar size without any fungicide treatment were collected from a greenhouse. The surfaces of the tomato fruits were sterilized with $0.5 \%$ sodium hypochlorite for $1 \mathrm{~min}$, rinsed three times with sterile distilled water, and then air dried. The surface-sterilized tomato fruits were sprayed with pydiflumetofen at 100, 200, and $300 \mathrm{mg} / \mathrm{liter}$, as well as the control fungicide boscalid at $300 \mathrm{mg} / \mathrm{liter}$, using a hand-held sprayer (Xinmeir Co., Ltd). The control fruits were sprayed with sterile distilled water. The spores of $B$. cinerea were obtained from 7-day-old colonies. Conidial suspensions were prepared with sterile water to a concentration of $1 \times 10^{5}$ spores $/ \mathrm{ml}$. A sterile fine needle was used to create a wound near the equator of the fruit, and $20 \mu l$ of the conidia suspension was inoculated into the wound. All of the treated fruits were kept for
3 days at $25^{\circ} \mathrm{C}$ and $80 \%$ relative humidity. The lesion size was then determined by measuring their two diameters at right angles to each other. Disease control efficacy was calculated as follows:

$$
\begin{aligned}
\operatorname{Efficacy}(\%) & =(\text { Lesion diameter in the water control } \\
& - \text { Lesion diameter in the treatment }) / \\
& \text { Lesion diameter in the water control } \times 100
\end{aligned}
$$

Three tomato fruits were used for each treatment, and all experiments were performed three times.

Correlation between pydiflumetofen and the other four SDHI fungicides. Seventy-two $B$. cinerea isolates that showed different levels of sensitivity to boscalid, fluopyram, isopyrazam, and benzovindiflupyr were selected from the isolates collected in 2018. The sensitivity of the 72 isolates to five SDHI fungicides (i.e., pydiflumetofen, boscalid, fluopyram, isopyrazam, and benzovindiflupyr) was determined by spore germination inhibition assays on YBA medium. Plates amended with equal volumes of methanol were used as controls. The concentrations of each fungicide were as follows: boscalid at $0,0.05,0.1,0.5,1,5,10$, and $50 \mathrm{mg}$ /liter; fluopyram at $0,0.005,0.01,0.05,0.1,0.5,1$, and $5 \mathrm{mg} / \mathrm{liter}$; isopyrazam at $0,0.005,0.01,0.05,0.1,0.5,1$, and $5 \mathrm{mg} / \mathrm{liter}$; and benzovindiflupyr at $0,0.005,0.01,0.05,0.1,0.5,1$, and $5 \mathrm{mg} /$ liter. Three replicate plates were used for each treatment, and all experiments were performed three times.

Greenhouse experiment. A greenhouse experiment was conducted in December 2018 on cucumber plants (cultivar Xintai Mici) in the village of Yuesihan $\left(36.77^{\circ} \mathrm{N}, 118.69^{\circ} \mathrm{E}\right)$, Shouguang City, Shandong Province, China. B. cinerea infection was prevalent in this greenhouse. Five spray application treatments were used: (i) water control; (ii, iii, and iv) pydiflumetofen at 100, 200, and $300 \mathrm{mg} /$ liter; and (v) control fungicide boscalid at $400 \mathrm{mg} / \mathrm{liter}$. All treatments were laid out in a randomized complete block design with three replications. Before spray application, 100 leaves with or without disease symptoms in each plot were marked with red strings, and the disease index was recorded. Spraying was performed twice at a $7-$ day interval. The efficacy and the disease index of the leaves were calculated 7 days after the last application according to a previously described method (Song et al. 2016).

Data analysis. $\mathrm{EC}_{50}$ values were calculated via regression of the percent relative growth against the $\log _{10}$ values of the fungicide concentrations. The sensitivity of $B$. cinerea isolates to pydiflumetofen was plotted against their sensitivity to fluopyram, benzovindiflupyr, isopyrazam, and boscalid. The $\mathrm{EC}_{50}$ values were transformed into $\log _{10}$ values, and correlations of $B$. cinerea sensitivities to pydiflumetofen and the other four SDHI fungicides were evaluated by Pearson correlation coefficients $(r)$. All of the data are represented as the mean \pm SE. Analysis of variance was carried out using Fisher's least significant difference test (SPSS version 13.0 for Windows) to determine statistically significant differences among the treatments $(P=0.05)$. The efficacy of the fungicides against gray mold was arcsine transformed prior to analysis.

\section{Results}

Sensitivity of $B$. cinerea to pydiflumetofen in vitro. Pydiflumetofen exhibited strong inhibitory activity against $B$. cinerea, and there

Table 1. Sensitivity of Botrytis cinerea to pydiflumetofen based on spore germination

\begin{tabular}{lccc}
\hline & & \multicolumn{2}{c}{ EC $_{\mathbf{5 0}}(\mathbf{m g} /$ liter $)$} \\
\cline { 3 - 4 } Year & Isolates $(\boldsymbol{n})$ & Range & Mean $\pm \mathbf{S E}^{\mathbf{z}}$ \\
\hline 2017 & 91 & $0.001-0.29$ & $0.06 \pm 0.01 \mathrm{a}$ \\
2018 & 93 & $0.001-0.72$ & $0.07 \pm 0.02 \mathrm{a}$ \\
2019 & 107 & $0.001-0.79$ & $0.05 \pm 0.02 \mathrm{a}$ \\
\hline
\end{tabular}

${ }^{\mathrm{z}}$ Mean values followed by the same letter in the columns were not significantly different by Fisher's least significant difference test at $P=0.05$. $\mathrm{EC}_{50}$, fungicide concentration resulting in a $50 \%$ inhibition compared with that of the control. 
was no difference in the sensitivity of $B$. cinerea to pydiflumetofen in 3 years (Table 1). The mean $\mathrm{EC}_{50}$ values of pydiflumetofen for spore germination in 2017,2018 , and 2019 were $0.06 \pm 0.01,0.07 \pm 0.02$, and $0.05 \pm 0.02 \mathrm{mg} /$ liter, respectively. The mean $\mathrm{EC}_{50}$ values of $B$. cinerea isolates collected from Laiwu and Liaocheng were significantly higher than those in the other eight regions (Supplementary Table S2). Among six crops, the mean $\mathrm{EC}_{50}$ value of $B$. cinerea isolates collected from eggplant was the highest (Supplementary Table $\mathrm{S} 3$ ). The baseline sensitivity of $B$. cinerea to pydiflumetofen was established using 234 single-spore isolates without any mutation. The frequency distribution of the $\mathrm{EC}_{50}$ values fit a unimodal curve (Fig. 1), with a mean value of $0.03 \pm 0.003 \mathrm{mg} / \mathrm{liter}$.

A total of 57 isolates with point mutations were detected from 291 isolates. The isolates carrying P225F, N230I, H272Y, and H272R mutations in the $S d h \mathrm{~B}$ subunit were associated with the less sensitivity of $B$. cinerea to SDHIs. The mean $\mathrm{EC}_{50}$ value of pydiflumetofen for the isolates with $\mathrm{P} 225 \mathrm{~F}, \mathrm{~N} 230 \mathrm{I}, \mathrm{H} 272 \mathrm{Y}$, and $\mathrm{H} 272 \mathrm{R}$ mutation was $0.31 \pm 0.09,0.07 \pm 0.02,0.29 \pm 0.05$, and $0.08 \pm 0.02 \mathrm{mg} / \mathrm{liter}$, respectively (Table 2). The RFs of $\mathrm{P} 225 \mathrm{~F}$ and $\mathrm{H} 272 \mathrm{Y}$ mutant isolates were the highest among the four mutation types, and they showed low to moderate levels of resistance to pydiflumetofen. The N230I and H272R mutant isolates showed low levels of resistance.

Efficacy of pydiflumetofen on detached tomato fruits. There was no significant difference in the efficacy of pydiflumetofen and boscalid against the sensitive isolates, and the efficacy of

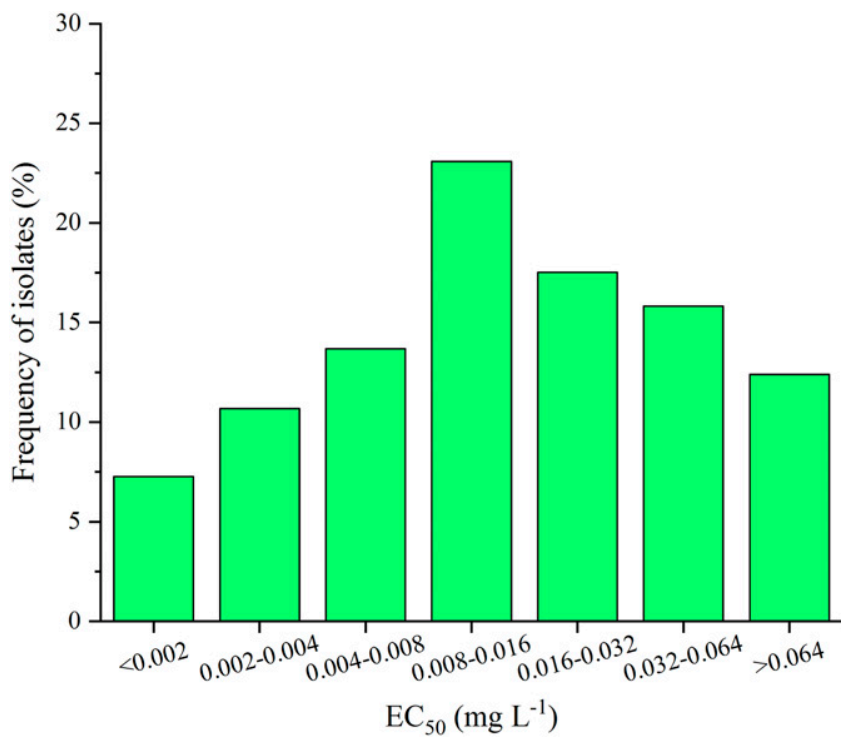

Fig. 1. Frequency distributions of $\mathrm{EC}_{50}$ values for pydiflumetofen against 234 Botrytis cinerea isolates collected in Shandong Province of China based on spore germination. $\mathrm{EC}_{50}$, fungicide concentration resulting in a $50 \%$ inhibition compared with that of the control.

Table 2. Sensitivity of Botrytis cinerea with four mutation types to pydiflumetofen based on spore germination

\begin{tabular}{lcccc}
\hline \multirow{2}{*}{$\begin{array}{l}\text { Mutation } \\
\text { type }\end{array}$} & Isolates $(\boldsymbol{n})$ & Range & Mean $\pm \mathbf{S E}^{\mathbf{y}}$ & Resistance factor $^{\mathbf{z}}$ \\
\cline { 3 - 5 } & 9 & $0.05-0.79$ & $0.31 \pm 0.09 \mathrm{a}$ & $1.6-26.3$ \\
$\mathrm{P} 225 \mathrm{~F}$ & 16 & $0.06-0.73$ & $0.29 \pm 0.05 \mathrm{a}$ & $2.0-24.3$ \\
$\mathrm{H} 272 \mathrm{Y}$ & 13 & $0.02-0.19$ & $0.07 \pm 0.02 \mathrm{~b}$ & $0.6-6.3$ \\
$\mathrm{~N} 230 \mathrm{I}$ & 19 & $0.01-0.39$ & $0.08 \pm 0.02 \mathrm{~b}$ & $0.3-13.0$ \\
$\mathrm{H} 272 \mathrm{R}$ & &
\end{tabular}

y Mean values followed by the same letter in the columns were not significantly different by Fisher's least significant difference test at $P=0.05$. $\mathrm{EC}_{50}$, fungicide concentration resulting in a 50\% inhibition compared with that of the control.

${ }^{\mathrm{z}}$ Resistance factors were calculated by dividing the $\mathrm{EC}_{50}$ value of resistant isolates by the mean $\mathrm{EC}_{50}$ value of sensitive isolates. pydiflumetofen and boscalid at $300 \mathrm{mg} /$ liter was 91.9 and $91.0 \%$, respectively (Fig. 2B). However, the efficacy of both fungicides was affected by the mutation types of the inoculated isolates (i.e., P225F, N230I, H272Y, and H272R). Comparatively, pydiflumetofen at 200 and $300 \mathrm{mg} /$ liter provided significantly higher efficacy than boscalid at $300 \mathrm{mg} /$ liter for mutant isolates (Fig. 2). Among four mutation types, $\mathrm{P} 255 \mathrm{~F}$ was the most difficult to control (Fig. 2C).

Correlation between pydiflumetofen and the other four SDHI fungicides. There was a positive correlation between pydiflumetofen and the other four SDHI fungicides (i.e., boscalid, fluopyram, isopyrazam, and benzovindiflupyr) (Fig. 3). The correlation coefficient between pydiflumetofen and isopyrazam was the largest, with an $r$ value of $0.62(P<0.05)$.

Greenhouse experiment. Although there was no significant difference in the efficacy of pydiflumetofen and boscalid against cucumber gray mold, the efficacy of pydiflumetofen at $300 \mathrm{mg} / \mathrm{liter}$ was much higher than that of boscalid at $400 \mathrm{mg} / \mathrm{liter}$. The efficacy of pydiflumetofen at $300 \mathrm{mg} /$ liter for leaves was $80.9 \%$, whereas that of boscalid at $400 \mathrm{mg} / \mathrm{liter}$ was $42.7 \%$ (Fig. 4).

\section{Discussion}

Currently, SDHI fungicides have become a research hotspot because of their wide control spectrum of diseases and unique mechanism of action (Xiong et al. 2017). Pydiflumetofen is a novel SDHI fungicide developed by Syngenta, and its activity on $B$. cinerea is largely unknown. It is well known that a positive correlation usually exists between SDHI fungicides (Avenot and Michailides 2010). Therefore, it is not only important to understand the activity of pydiflumetofen on SDHI-sensitive isolates of $B$. cinerea, but it is also essential to evaluate the efficacy of pydiflumetofen on SDHI-resistant isolates.

Consistent with the reported sensitivity of $B$. cinerea to other SDHI fungicides (He et al. 2018a; Song et al. 2016; Veloukas and Karaoglanidis 2012), the new SDHI fungicide pydiflumetofen had strong inhibitory activity on spore germination of $B$. cinerea. This study found that there was no difference in the sensitivity of $B$. cinerea to pydiflumetofen among the 3 years $(2017,2018$, and 2019). This finding indicated that the inhibitory activity of pydiflumetofen against $B$. cinerea was very stable. According to China Pesticide Information Software, we found that conventional fungicides such as carbendazim, boscalid, procymidone, and iprodione accounted for a large percentage of the registered fungicides for controlling gray mold. However, $B$. cinerea has been severely resistant to benzimidazoles and dicarboximides (Liu et al. 2016; Rupp et al. 2017). Because of the unique mechanism of action, pydiflumetofen shows no cross-resistance with conventional fungicides such as benzimidazoles and dicarboximides (Duan et al. 2019). Therefore, we believed that pydiflumetofen has a potential application for management of benzimidazole- and dicarboximide-resistant isolates. What is more, pydiflumetofen has great control for SDHI-resistant isolates of B. cinerea. It is well known that boscalid is the most commonly used SDHI fungicide for controlling gray mold in China. Frequent use of boscalid has led to the emergence of resistant isolates of $B$. cinerea in the field (Gutiérrez-Alonso et al. 2017). We found that the isolates carrying P225F, N230I, H272Y, and H272R mutations in the $S d h \mathrm{~B}$ subunit were responsible for the less sensitivity of $B$. cinerea to boscalid in Shandong Province. These resistant isolates have led to the failure of boscalid for controlling gray mold at registered concentrations, which was confirmed by the detached tomato assay in the current study. We also found that the efficacy of pydiflumetofen was superior to boscalid against gray mold in both the detached tomato assay and the greenhouse experiment. This finding suggested that pydiflumetofen could provide effective management of the current resistance to SDHI fungicides. Previous studies found that the protective effect of pydiflumetofen is better than its curative effect (Duan et al. 2019), which is consistent with other SDHI fungicides (He et al. 2018a; Song et al. 2016; Veloukas and Karaoglanidis 2012). Therefore, pydiflumetofen is recommended for use in the initial stage of gray mold occurrence.

It is important to perform resistance risk assessment of new fungicides before they enter the market. Therefore, baseline sensitivity of pathogenic fungi to new fungicides should be established for 

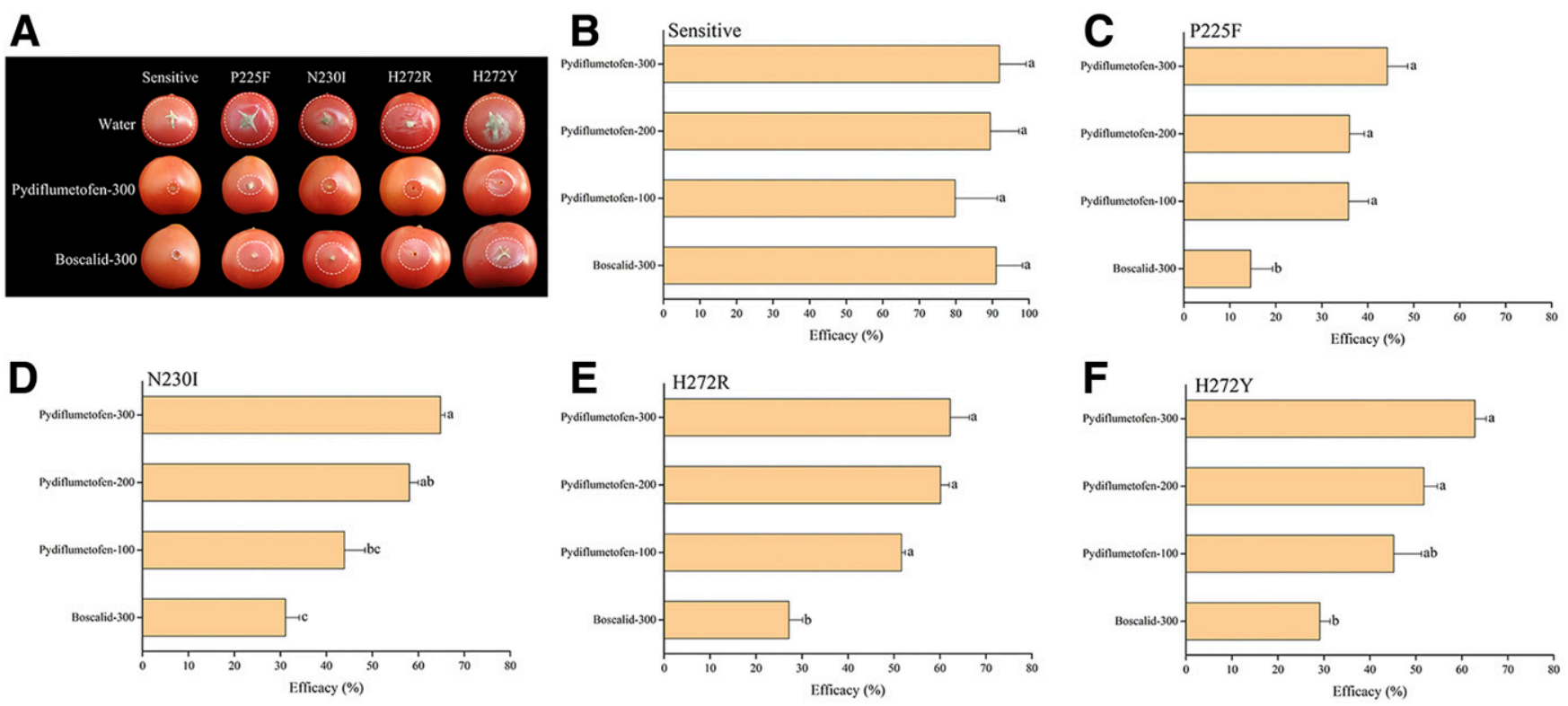

Fig. 2. Efficacy of pydiflumetofen on detached tomatoes inoculated with different phenotypes of Botrytis cinerea. A, visible symptoms; B, sensitive; C, P225F; D, N230l; E, H272R; and $\mathrm{F}, \mathrm{H} 272 \mathrm{Y}$ ). Mean values followed by the same letter in the columns were not significantly different by Fisher's least significant difference test at $P=0.05$.
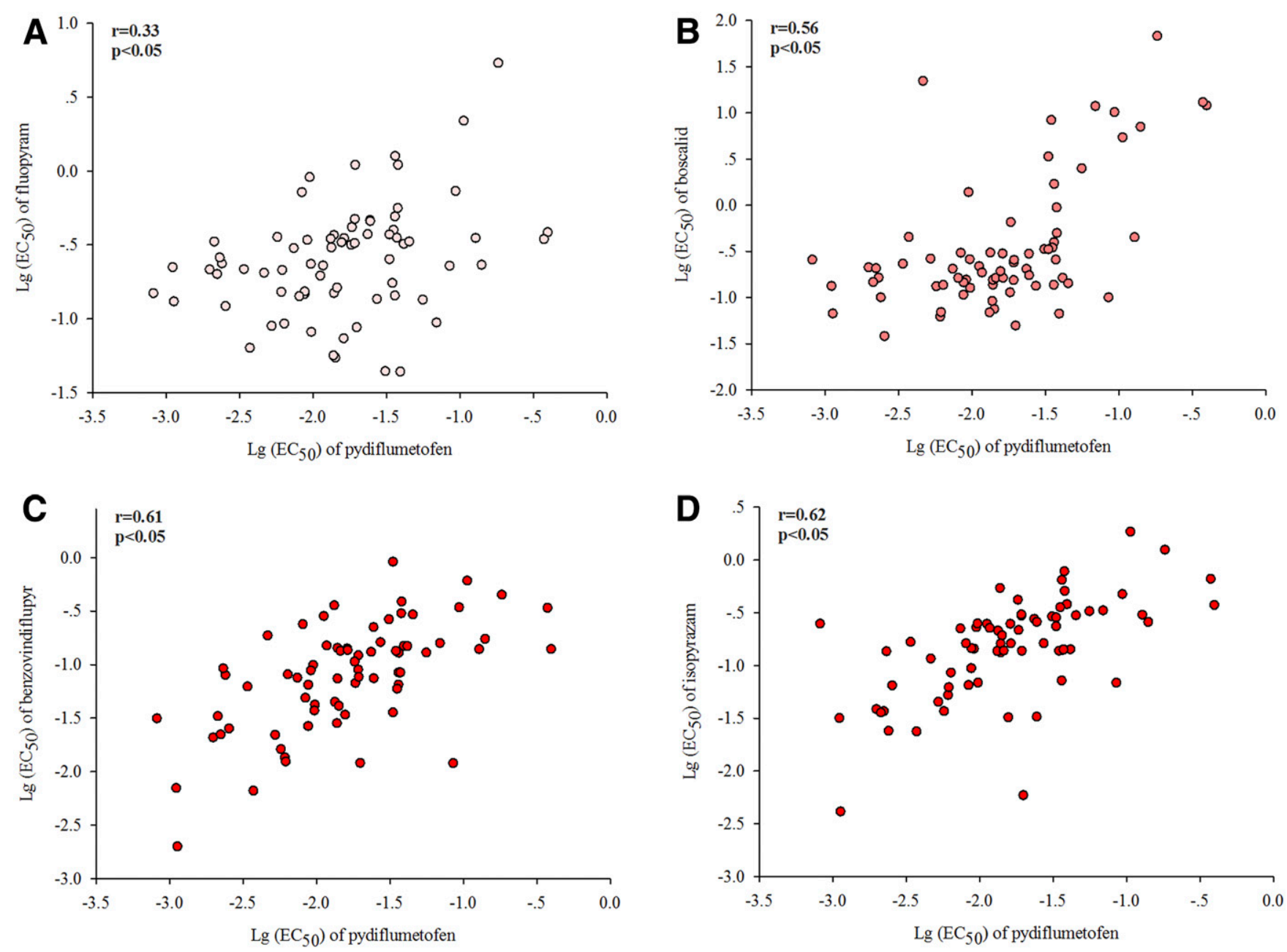

Fig. 3. Correlation between pydiflumetofen and $\mathbf{A}$, fluopyram, $\mathbf{B}$, boscalid, $\mathbf{C}$, benzovindiflupyr, and $\mathbf{D}$, isopyrazam. $\mathrm{EC}_{50}$, fungicide concentration resulting in a $50 \%$ inhibition compared with that of the control. 
subsequent monitoring of fungal resistance (Huang et al. 2019). After establishing the sensitivity baseline of $B$. cinerea to pydiflumetofen, we evaluated the resistance level of $B$. cinerea isolates with four mutation types to pydiflumetofen. Many studies reported that mutation types $\mathrm{P} 225 \mathrm{~F}$ and $\mathrm{N} 230 \mathrm{I}$ are responsible for the resistance of $\mathrm{B}$. cinerea to boscalid, fluopyram, and isopyrazam; H272R and H272Y mutant isolates are moderately resistant to boscalid and have low resistance to isopyrazam but are sensitive to fluopyram (Lalève et al. 2014; Veloukas et al. 2013). We found that P225F and H272Y mutant isolates showed low to moderate levels of resistance to pydiflumetofen, and H272R and N230I mutant isolates showed a low level of resistance. In the current study, we first proved that the reduced sensitivity of $B$. cinerea with four mutation types to pydiflumetofen was mainly attributable to the positive correlation of pydiflumetofen with the other four SDHI fungicides (i.e., boscalid, fluopyram, isopyrazam, and benzovindiflupyr). As we know, once the structure of the target protein changes, its binding energy to the fungicide compound generally decreases, thereby resulting in fungicide resistance (Kretschmer et al. 2009). Previous studies reported that pyrazole is more potent than pyridine in combination with the target protein, and introducing the fluorine atom into the compound structure increases the binding energy of the compound to the target protein (Fraaije et al. 2012; Scalliet et al. 2012). Our results showed that $B$. cinerea isolates with four mutation types were more sensitive to pydiflumetofen than boscalid. We speculate that maybe the structural characteristics of pyrazole and fluorine atoms on pydiflumetofen (Supplementary Fig. S1) result in the sensitivity difference between pydiflumetofen and boscalid. In the current study, the P225F mutation was less sensitive to pydiflumetofen than other mutation types, and the resistance frequency of the P255F mutation was highly detected in the cities of Laiwu and Liaocheng in Shandong Province. Therefore, although pydiflumetofen could effectively manage the current resistance of SDHI fungicides, it still should be used with caution in specific areas.

In conclusion, pydiflumetofen is currently effective for controlling gray mold, and it can be used as a substitute for boscalid to manage gray mold in the field. However, current SDHI-resistant isolates of $B$. cinerea showed reduced sensitivity to pydiflumetofen, although the resistance to pydiflumetofen was only at low to moderate levels. SDHI fungicides are marked with a medium to high resistance risk, which makes us aware of the importance of delaying the development of resistance (Fungicide Resistance Action Committee 2018). In the future, the sensitivity of $B$. cinerea to pydiflumetofen should be continuously monitored to effectively adjust the application schedule. In addition, precise application techniques can decrease the selective pressure of fungicides, thereby delaying the development of fungal resistance (He et al. 2018b). Therefore, future research should focus on exploring more efficient application techniques.

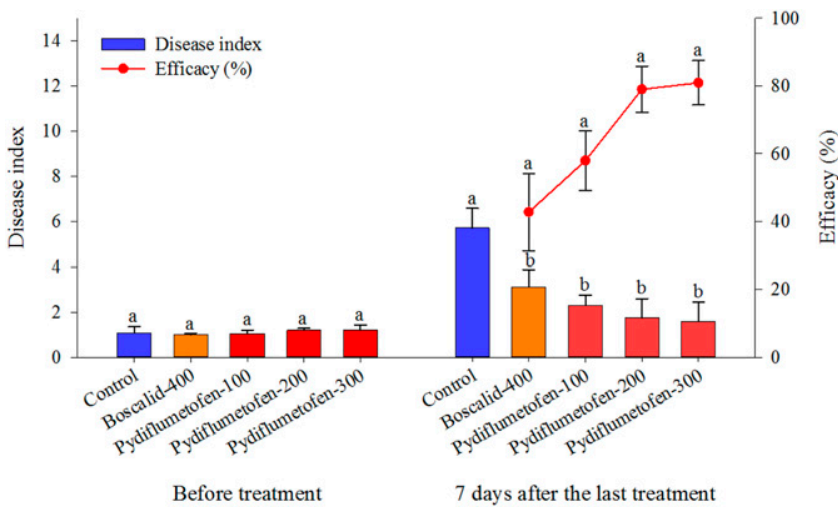

Fig. 4. Efficacy of pydiflumetofen and boscalid on cucumber leaves in the greenhouse experiment. Mean values followed by the same letter in the columns were not significantly different by Fisher's least significant difference test at $P=0.05$.

\section{Literature Cited}

Avenot, H. F., and Michailides, T. J. 2010. Progress in understanding molecular mechanisms and evolution of resistance to succinate dehydrogenase inhibiting (SDHI) fungicides in phytopathogenic fungi. Crop Prot. 29:643-651.

Bardas, G. A., Veloukas, T., Koutita, O., and Karaoglanidis, G. S. 2010. Multiple resistance of Botrytis cinerea from kiwifruit to SDHIs, QoIs and fungicides of other chemical groups. Pest Manag. Sci. 66:967-973.

Buxton, K., Harp, T., Tally, A., and Mclean, H. 2016. Adepidyn: A new fungicide active ingredient for control of foliar diseases. Phytopathology 106:61.

Duan, Y. B., Xiu, Q., Li, H. R., Li, T., Wang, J. X., and Zhou, M. G. 2019. Pharmacological characteristics and control efficacy of a novel SDHI fungicide pydiflumetofen against Sclerotinia sclerotiorum. Plant Dis. 103:77-82.

Fan, F., Hamada, M. S., Li, N., Li, G. Q., and Luo, C. X. 2017. Multiple fungicide resistance in Botrytis cinerea from greenhouse strawberries in Hubei Province, China. Plant Dis. 101:601-606.

Fernández-Ortuño, D., Chen, F., and Schnabel, G. 2012. Resistance to pyraclostrobin and boscalid in Botrytis cinerea isolates from strawberry fields in the Carolinas. Plant Dis. 96:1198-1203.

Fraaije, B. A., Bayon, C., Atkins, S., Cools, H. J., Lucas, J. A., and Fraaije, M. W. 2012. Risk assessment studies on succinate dehydrogenase inhibitors, the new weapons in the battle to control Septoria leaf blotch in wheat. Mol. Plant Pathol. 13:263-275.

Fungicide Resistance Action Committee. 2018. FRAC Code List 2018: Fungicide Sorted by Mode of Action. https://web.archive.org/web/20180403125706/https:// www.frac.info/docs/default-source/publications/frac-code-list/frac_code_ list_2018-final.pdf

Gutiérrez-Alonso, O., Hawkins, N. J., Cools, H. J., Shaw, M. W., and Fraaije, B. A 2017. Dose-dependent selection drives lineage replacement during the experimental evolution of SDHI fungicide resistance in Zymoseptoria tritici. Evol. Appl. 10:1055-1066.

He, L., Cui, K., Song, Y., Mu, W., and Liu, F. 2018a. High-efficiency control of gray mold by the novel SDHI fungicide benzovindiflupyr combined with a reasonable application approach of dipping flower. J. Agric. Food Chem. 66:6692-6698.

He, L., Cui, K., Song, Y., Zhang, Z., Li, B., Mu, W., and Liu, F. 2018b. A precisely targeted application strategy of dipping young cucumber fruit in fungicide to control cucumber gray mold. Pest Manag. Sci. 74:2432-2437.

Hou, Y. P., Mao, X. W., Wang, J. X., Zhan, S. W., and Zhou, M. G. 2017. Sensitivity of Fusarium asiaticum to a novel succinate dehydrogenase inhibitor fungicide pydiflumetofen. Crop Prot. 96:237-244.

Hu, M., Fernández-Ortuño, D., and Schnabel, G. 2016. Monitoring resistance to SDHI fungicides in Botrytis cinerea from strawberry fields. Plant Dis. 100: 959-965.

Huang, X. P., Song, Y. F., Li, B. X., Mu, W., and Liu, F. 2019. Baseline sensitivity of isopyrazam against Sclerotinia sclerotiorum and its efficacy for the control of sclerotinia stem rot in vegetables. Crop Prot. 122:42-48.

Kim, Y. K., and Xiao, C. L. 2010. Resistance to pyraclostrobin and boscalid in populations of Botrytis cinerea from stored apples in Washington state. Plant Dis. 94:604-612.

Kretschmer, M., Leroch, M., Mosbach, A., Walker, A. S., Fillinger, S., Mernke, D., Schoonbeek, H. J., Pradier, J. M., Leroux, P., De Waard, M.A., and Hahn, M. 2009. Fungicide-driven evolution and molecular basis of multidrug resistance in field populations of the grey mould fungus Botrytis cinerea. PLoS Pathog. 5:e1000696.

Lalève, A., Gamet, S., Walker, A. S., Debieu, D., Toquin, V., and Fillinger, S. 2014. Site-directed mutagenesis of the P225, N230 and H272 residues of succinate dehydrogenase subunit B from Botrytis cinerea highlights different roles in enzyme activity and inhibitor binding. Environ. Microbiol. 16: 2253-2266.

Leroux, P., Gredt, M., Leroch, M., and Walker, A. S. 2010. Exploring mechanisms of resistance to respiratory inhibitors in field strains of Botrytis cinerea, the causal agent of gray mold. Appl. Environ. Microbiol. 76:6615-6630.

Liu, S., Che, Z., and Chen, G. 2016. Multiple-fungicide resistance to carbendazim, diethofencarb, procymidone, and pyrimethanil in field isolates of Botrytis cinerea from tomato in Henan Province, china. Crop Prot. 84:56-61.

Rupp, S., Weber, R. W., Rieger, D., Detzel, P., and Hahn, M. 2017. Spread of Botrytis cinerea strains with multiple fungicide resistance in German horticulture. Front. Microbiol. 7:2075-2087.

Scalliet, G., Bowler, J., Luksch, T., Kirchhofer-Allan, L., Steinhauer, D., and Ward, K 2012. Mutagenesis and functional studies with succinate dehydrogenase inhibitors in the wheat pathogen Mycosphaerella graminicola. PLoS One 7:e35429.

Song, Y., Zhang, Z., Chen, L., He, L., Lu, H., and Ren, Y. 2016. Baseline sensitivity of Botrytis cinerea to the succinate dehydrogenase inhibitor isopyrazam and efficacy of this fungicide. Plant Dis. 100:1314-1320.

Veloukas, T., and Karaoglanidis, G. S. 2012. Biological activity of the succinate dehydrogenase inhibitor fluopyram against Botrytis cinerea and fungal baseline sensitivity. Pest Manag. Sci. 68:858-864.

Veloukas, T., Leroch, M., Hahn, M., and Karaoglanidis, G. S. 2011. Detection and molecular characterization of boscalid-resistant Botrytis cinerea isolates from strawberry. Plant Dis. 95:1302-1307.

Veloukas, T., Markoglou, A. N., and Karaoglanidis, G. S. 2013. Differential effect of SdhB gene mutations on the sensitivity to SDHI fungicides in Botrytis cinerea. Plant Dis. 97:118-122. 
Wang, Y., Duan, Y., Wang, J., and Zhou, M. 2015. A new point mutation in the iron-sulfur subunit of succinate dehydrogenase confers resistance to boscalid in Sclerotinia sclerotiorum. Mol. Plant Pathol. 16:653-661.

Xiong, L., Hua, L., Jiang, L. N., Ge, J. M., Yang, W. C., Zhu, X. L., and Yang, G. F. 2017. Structure-based discovery of potential fungicide as succinate ubiquinone oxidoreductase inhibitors. J. Agric. Food Chem. 65:1021-1029.
Yin, Y. N., Kim, Y. K., and Xiao, C. L. 2011. Molecular characterization of boscalid resistance in field isolates of Botrytis cinerea from apple. Phytopathology 101:986-995.

Zheng, Y., Yang, Y., Liu, C., Chen, L., Sheng, J., and Shen, L. 2015. Inhibition of SLMPK1, SLMPK2, and SLMPK3 disrupts defense signaling pathways and enhances tomato fruit susceptibility to Botrytis cinerea. J. Agric. Food Chem. 63:5509-5517. 